\title{
Numerical heat transfer during Herschel-Bulkley fluid natural convection by CVFEM
}

\author{
Diego A. $\operatorname{Vasco}^{1 *}$, Carlos Salinas ${ }^{2}$, Nelson Moraga ${ }^{3}$, Roberto Lemus-Mondaca ${ }^{4}$ \\ ${ }^{1}$ Departamento de Ingeniería Mecánica. Universidad de Santiago de Chile. Av. Lib. Bernardo O’Higgins 3363, Santiago, Chile \\ ${ }^{2}$ Departamento de Ingeniería Mecánica. Universidad del Bío-Bío. Av. Collao 1202, Concepción, Chile \\ ${ }^{3}$ Departamento de Ingeniería Mecánica. Universidad de La Serena. Benavente 980, La Serena, Chile \\ ${ }^{4}$ Departamento de Ciencia de los Alimentos y Tecnología Química, Universidad de Chile. Santos Dumont 964, Independencia, \\ Santiago, Chile
}

Corresponding Author Email: diego.vascoc@usach.cl

https://doi.org/10.18280/ijht.360221

Received: 19 November 2017

Accepted: 9 April 2018

\section{Keywords:}

free convection, heat transfer, non-

Newtonian fluid, CVFEM

\begin{abstract}
Numerical prediction of heat transfer by natural convection of a Herschel-Bulkley nonNewtonian fluid inside a square cavity has been computationally analyzed. Unsteady 2D fluid mechanics and heat transfer were described in terms of the non-linear coupled continuity, momentum and heat equations. These equations were solved by the control volume finite element method (CVFEM) with Gauss-Seidel/System Over-Relaxation coupling algorithm. The effect of the $R a, P r, B n$ and the rheological behavior index (n) on the non-Newtonian fluid thermal and momentum behavior were studied. The non-Newtonian fluid flow was described by the rheological model of Herschel-Bulkley. Results for the streamlines and isotherms along the enclosure walls are presented. It was found that the effect of the $\mathrm{Pr}$ and $\mathrm{Bn}$ is more important when the $\mathrm{Ra}$ is lower $\left(10^{3}\right)$. In addition, the behavior index had a significant effect on the CPU time for the different studied cases.
\end{abstract}

\section{INTRODUCTION}

Processes involving shear thinning non-Newtonian fluids are of great importance in several biological applications, chemical and food industry [1-3]. For instance, thermal processing of pulps and fruit juices is interesting in food industry. Several studies have demonstrated that rheological properties of fruit juices are well described by shear-thinning Non-Newtonian models such as Power-law [4-5], HershelBulkley [6], Bingham and Casson [7]. Most of foregoing mentioned experimental studies consider the effect of solutes concentration, water content, and temperature in the rheological parameters.

Natural convection involving Newtonian and nonNewtonian fluids has been broadly investigated. Both experimental and numerical studies have been made; hence benchmark reliable results are available for two and threedimensional domains [8-11]. Nevertheless due to experimental difficulties, to the best knowledge of the authors, only numerical studies have been conducted with NonNewtonian fluids in both 2D and 3D domains [12-15]. The authors, Anwar-Hossain and Reddy-Gorla [16], studied the flow of a power law Non-Newtonian fluid past an isothermal vertical slotted surface. They found that as the behavior index increase, both the friction factor and the Nusselt number increased. Also with a power law Non-Newtonian fluid, a study of the Rayleigh-Bénard problem confirmed the previous observation respect the behavior index of shear-thinning fluids $(0<\mathrm{n}<1)$. Nevertheless, for shear-thickening fluids $(\mathrm{n}>1)$, the opposite effect was observed by Lamsaadi and Naïmi [17].

An analytical and numerical study by the finite difference method was performed in a differentially heated sided walls of a rectangular enclosure filled with a power law fluid [18]. It was found that at high Prandtl numbers natural convection is mainly controlled by the flow behavior index and the Rayleigh number. The same physical situation was studied by Vola et al. [19] and Turan et al. [13] with a square enclosure filled with a yield stress fluid obeying the Bingham model. In the former work, through domain discretization by low order finite elements, the yield stress effect on temperature and velocity distribution was studied. The obtained results show that at higher yield stress values the apparition of rigid zones modified the vortices position and the temperature distribution turns out to be controlled by thermal diffusion. Meanwhile, in the latter work, the Fluent v.6 commercial software was used to solve the coupled conservation equation of mass, momentum, and energy.

Respect to the rheological properties of the non-Newtonian fluid, for high values of the Bingham number, no significant flow is induced within the enclosure. It is known that in most buoyancy-driven motions, the flow is slow due to moderate temperature gradients [20]. Kumar and Bhattacharya [21] predicted unsteady natural convection and heat transfer during sterilization of a temperature dependent power-law NonNewtonian fluid using the finite volume method. The authors found out that there was not a fixed point, which could be called the coldest point at all times. Certainly, the rheological properties have a significant influence on the transient processes [22]. Dean et al. [23] presented a wide review of results and numerical methods concerning with forced convection for Bingham plastic fluids.

The control volume finite-element method (CVFEM) developed by Baliga and Patankar [24] holds the topological characteristics of the finite element method (FEM) and the conservative properties of the finite volume method (FVM). The control volumes are made up of several finite elements 
and each element contributes to three different control volumes. The way how the control volumes are built allows that the fluxes through the boundaries can be calculated through interpolation techniques developed in FEM [25]. Thus, CVFEM considers linear orthotropic variations of the properties and independent variables within the finite element, considering the discrete variable centered on the control volume. The numerical approach leads to the formulation of linear algebraic equations systems that are solved through iterative and direct methods like Gauss-Seidel with SOR and Gauss Elimination [26]. The CVFEM method has been successfully used in applications with thermofluids [24], moving boundary problems [27-28], solid mechanics [25], and functionally graded materials [29].

The main objective of the present work is to examine the natural convection heat transfer characteristics of a nonNewtonian fluid filled in a square cavity based on the Herschel-Bulkley fluid model and solved by finite element/control volume method (CVFEM), for different Ra, $B n$ and $P r$ numbers and behavior index. A comparison of the behavior of streamlines, pressures, velocity profiles, isotherms, $N u$ numbers in the cavity, caused by the variation in the governing parameters, is presented.

\section{PHYSICAL SITUATION}

The natural convection of the non-Newtonian fluid inside a square domain considered in this paper is shown in Figure 1. The problem is assumed to be two-dimensional. The dimensions of the enclosure are equal and denoted by L. The left and right vertical walls are differentially heated at isothermal temperatures of $T c$ and $T h$, respectively, whereas the remaining walls are considered adiabatic.

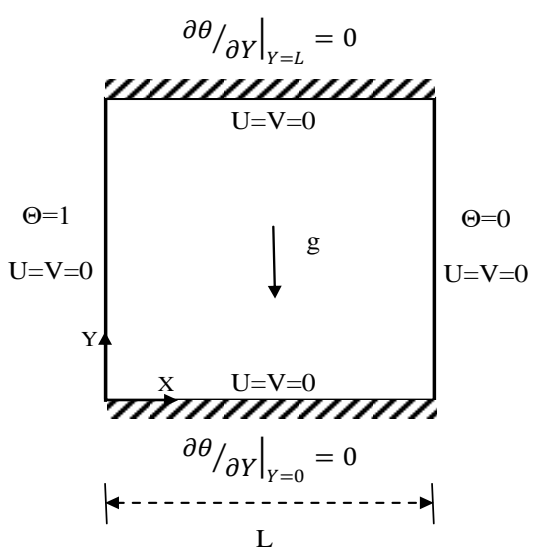

Figure 1. Physical 2D domain of natural convection heat transfer process

\section{MATHEMATICAL MODEL}

\subsection{Governing equations}

The governing equations are formulated for an incompressible non-Newtonian fluid with constant thermophysical properties. Density for the gravitational term in the y-momentum equation is assumed to have linear dependence of temperature changes according to the Boussinesq approximation. Therefore, dimensional equations for the natural convection of a Non-Newtonian fluid in a twodimensional square cavity are described by:

Mass conservation equation:

$\frac{\partial u}{\partial x}+\frac{\partial v}{\partial y}=0$

Momentum conservation equations:

$\frac{\partial(\rho u)}{\partial t}+u \frac{\partial(\rho u)}{\partial x}+v \frac{\partial(\rho u)}{\partial y}-\nabla(\eta(\dot{\gamma}) \nabla u)+\frac{\partial p}{\partial x}=0$
$\frac{\partial(\rho v)}{\partial t}+u \frac{\partial(\rho v)}{\partial x}+v \frac{\partial(\rho v)}{\partial y}-\nabla(\eta(\dot{\gamma}) \nabla v)-\rho g \beta\left(T-T_{r e f}\right)+\frac{\partial p}{\partial y}=0$

Energy conservation equation:

$\frac{\partial(\rho C p T)}{\partial t}+u \frac{\partial(\rho C p T)}{\partial x}+v \frac{\partial(\rho C p T)}{\partial y}-\nabla(K \nabla T)=0$

The non-dimensional version of the governing equations is achieved according to the following dimensionless parameters:

$X=\frac{x}{L} ; Y=\frac{y}{L}$

$\theta=\frac{T-T_{H}}{T_{H}-T_{C}} ; P=\frac{p L}{\eta_{0} \sqrt{g \beta \Delta T L}} ; U=\frac{u}{\sqrt{g \beta \Delta T L}} ; \quad V=\frac{v}{\sqrt{g \beta \Delta T L}}$

$\mathrm{H}=\frac{\eta}{\eta_{o}} ; \dot{\Gamma}=\frac{L}{\sqrt{g \beta \Delta T L}} \dot{\gamma}$

The non-dimensional governing equations are then expressed as:

Mass conservation equation:

$\frac{\partial U}{\partial X}+\frac{\partial V}{\partial Y}=0$

Momentum conservation equations:

$\sqrt{\frac{R a}{P r}}\left(U \frac{\partial U}{\partial X}+V \frac{\partial U}{\partial Y}\right)-\nabla(\mathrm{H}(\dot{\Gamma}) \nabla U)+\frac{\partial P}{\partial X}=0$

$\sqrt{\frac{R a}{P r}}\left(U \frac{\partial V}{\partial X}+V \frac{\partial V}{\partial Y}\right)-\nabla(\mathrm{H}(\dot{\Gamma}) \nabla V)-\sqrt{\frac{R a}{P r}} \theta+\frac{\partial P}{\partial Y}=0$

Energy conservation equation:

$\sqrt{R a \cdot \operatorname{Pr}}\left(U \frac{\partial \theta}{\partial X}+V \frac{\partial \theta}{\partial Y}\right)-\nabla(\nabla \theta)=0$

The non-dimensional parameters obtained are:

$R a=\frac{g \beta\left(T_{H}-T_{C}\right) L^{3}}{\eta_{0} \alpha}$

$\operatorname{Pr}=\frac{\eta_{0} C p}{k}$

\subsection{Herschel-Bulkley fluid model}

This model is characterized by showing no deformation up to a certain level of stress, however above this yield stress the material flows readily [30]. Then, Herschel-Bulkley model in tensorial form can be expressed as:

$\overline{\bar{\tau}}=\left(\frac{\tau_{y}}{\dot{\gamma}}+k \dot{\gamma}^{n-1}\right) \overline{\bar{\gamma}} ;$ for $|\tau|>\tau_{y}$ 
$\overline{\bar{\gamma}}=0 ;$ for $|\tau| \leq \tau_{y}$

It could be noticed that for $n=1$ the Herschel-Bulkley model is reduced to the Bingham model and for $\tau_{y}=0$ the Ostwald-De Waele power-law model is obtained.

Papanastasiou [31] proposed an alternative approach to overcome the issue that represents yield stress, avoiding the discontinuity in the flow curve due to the incorporation of the yield criterion. The modification involves the incorporation of an exponential term, thereby permitting the use of one equation for the entire flow curve, before and after yield. A modified Herschel-Bulkley model to approximate the rheological behavior is given by:

$\mathrm{H}(\dot{\Gamma})=\dot{\Gamma}^{n-1}+\frac{B n}{\dot{\Gamma}}[1-\exp (-M \dot{\Gamma})]$

$B n=\frac{\tau_{y}}{\eta_{0}} \sqrt{\frac{L}{g \beta \Delta T}}$

$M=m \sqrt{\frac{g \beta \Delta T}{L}}$

In addition, the $m$ parameter when increasing the Papanastasiou modified Herschel-Bulkley model is approached the Herschel-Bulkley model at low shear rates. The bi-viscosity models and Papanastasiou's modification are empirical improvements designed primarily to afford a convenient viscoplastic constitutive equation for numerical simulations [32].

\subsection{Boundary and initial conditions}

The governing equations are complemented through the implementation of the boundary and initial conditions. Noslippery boundary conditions for the velocities at the walls are settled. Heat transfer boundary conditions include uniform hot and cold temperature on the vertical left and right walls, respectively, along adiabatic condition on the other walls. In addition, the initial condition for velocities, pressure and temperature inside the cavity corresponds to null values.

$$
\begin{aligned}
& \theta(0, Y)=1 ; \theta(1, Y)=0 \\
& \left.\frac{\partial \theta}{\partial Y}\right|_{Y=0}=0 ;\left.\quad \frac{\partial \theta}{\partial Y}\right|_{Y=1}=0
\end{aligned}
$$

\section{CVFEM IMPLEMENTATION}

The governing equations could be represented by a general expression (17a), which contains the transient, convective, diffusive and source terms:

$\frac{\partial \phi}{\partial t}+\nabla \vec{V} \phi-\nabla(\Gamma \nabla \phi)-\mathrm{F}=0$

This expression could be represented as well in terms of flux divergence

$(\vec{J}): \frac{\partial \phi}{\partial t}+\nabla(\vec{J})-\mathrm{F}=0$

with:

$\vec{J}=\vec{v} \phi-\Gamma \nabla \phi$
Depending on the transported quantity, terms in equation (17a) are modified according to Table 1 . In the same way that finite volume method, the starting-point of this method is the integration of the generalized transport equation (17b) over a control volume $V^{n}$ with a contour area $S^{n}(n=1, \ldots, N)$.

Table 1. Coefficients of the generalized transport equation (18) for each variable

\begin{tabular}{cccc}
\hline$\phi$ & $\rho$ & $\Gamma$ & $\mathrm{S}$ \\
\hline$U$ & $\sqrt{\frac{R a}{P r}}$ & $\mathrm{H}(\dot{\Gamma})$ & $\partial P / \partial X$ \\
$V$ & $\sqrt{\frac{R a}{P r}}$ & $\mathrm{H}(\dot{\Gamma})$ & $\partial P / \partial Y+\sqrt{\frac{R a}{P r}} \theta$ \\
$\theta$ & $\sqrt{R a \cdot P r}$ & 1 & - \\
\hline
\end{tabular}

Applying the Green Theorem:

$\int_{V^{n}} \frac{\partial \phi}{\partial t} d V+\int_{s^{n}}((\phi v-\Gamma \nabla \phi) \cdot \widehat{\mathbf{n}}) d s-\int_{V^{n}} F d V=0$

where each $V^{n}$ is made up by partial contributions of the finite elements $V_{e}(e=1, . ., E)$ (Figure 2):

$V=\sum_{n=1}^{N} V^{l}$

with

$V^{l}=\sum_{e=1}^{E} V_{e} \quad$ and $\quad S^{n}=\sum_{e=1}^{E} \underbrace{V^{n} \cap V_{e}}_{S_{e}^{n}}$

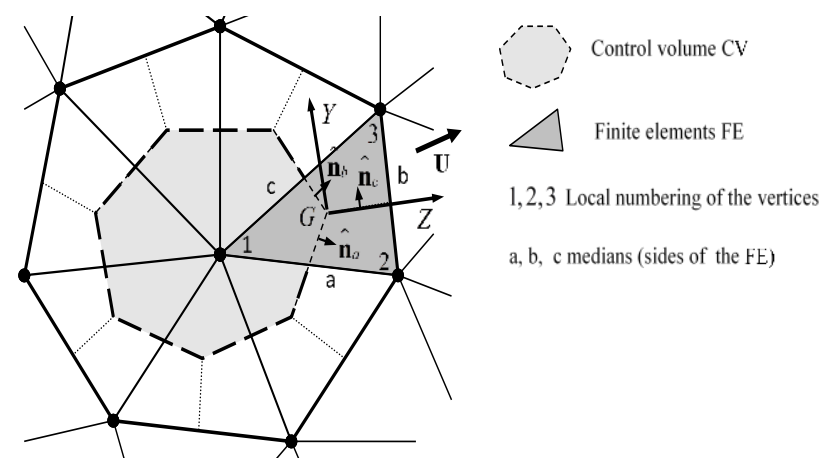

Figure 2. Local system of coordinates $Y / Z$ defined in the center of each control volume conformed by finite elements

In the same way each $S_{e}^{n}$ is built by two segments. For the control volume CV centered on the local vertex 1 (Figure 2), $S_{k}^{l}=S_{k a}^{l}+S_{k c}^{l}$, with normal vectors $\left(n_{a}, n_{c}\right)$ to the segments between the medians of the FE $(\mathrm{a}, \mathrm{c})$ to the centroid $(\mathrm{G})$. The integration of the convective and diffusive terms of the equation (17a) requires a function, which represents the distribution of $\phi$ over each FE. Generally, a linear function (20a) is adopted for the diffusion term and an exponential function based on local coordinates (20b) for the convective term:

$\phi(x, y)=A x+B y+C$

$\phi(Z, \zeta)=A Z+B \zeta+C$

In both cases, the coefficients $\mathrm{A}, \mathrm{B}$, and $\mathrm{C}$ are defined 
according to the nodal values of the dependent variable $\phi$ and the coordinates of the vertices of the FE (local nodes 1,2 and $3)$. The non-linear interpolation of the convective term requires first the definition of a local system of coordinates $(Z, \zeta)$ for each FE, where the Z-axis is parallel to the velocity vector $v_{i}$ with module $\mathrm{U}$ (Figure 2). The new $\mathrm{Z}$-values are obtained through an exponential function of the original coordinate values $\mathrm{X}$ and the Peclet number:

$Z=\frac{\Gamma}{\rho U}\left[\exp \left(\frac{P e\left(X-X_{\max }\right)}{X_{\max }-X_{\min }}\right)-1\right]$

The approach above along with an implicit backward Eulerian scheme for the transient term, allows the representation of the equation (17a) in discrete terms for each node $p$ of the domain:

$a_{p} \phi_{p}=\sum_{n b} a_{n b} \phi_{n b}+b_{p}$

The obtained system of linear algebraic equations builds up sparse matrices with null values distributed randomly. To avoid unnecessary operations with null values, the non-null values of the sparse matrices and its positions are stored in two vectors through the compressed row storage (CRS) scheme. The resulting systems of equations are solved through the iterative method Gauss-Seidel with SOR (System OverRelaxation). For more details about the computational implementation see the work of Salinas et al. [27]. The calculations were accomplished by using a personal computer (Intel Core I7, 3.40GHz; 8 GB memory). The Intel Fortran compiler IFORT under Linux platform was used for the compilation of the Fortran 90 source codes. All variables were defined as double-precision floating-point data types. The convergence criteria applied to stop the velocity and temperature calculations was based in a maximum value for the difference in the calculated value at two successive iterations, defined as follows:

$\left|\phi_{i, j, k}^{k}-\phi_{i, j, k}^{k-1}\right| \leq 10^{-6}$

\section{SCALING ANALYSIS}

Following the same procedure performed by Turan et al. [13] for scaling analysis of natural convection of Bingham fluids, the effects of $R a, P r, B n$ and $n$ on the Nusselt number for a Herschel-Bulkley fluid are analyzed. The wall heat flux $\mathrm{q}$ is scaled as:

$q \sim k \frac{\Delta T}{\delta_{t h}} \sim h \Delta T$

which gives rise to the following expression:

$N u \sim \frac{h L}{k} \sim \frac{L}{\delta_{t h}} \quad$ or $N u \sim \frac{L}{\delta} \mathrm{g}(\operatorname{Pr}, B n, n)$

where the thermal boundary layer thickness $\delta_{t h}$ is related to the hydrodynamic boundary layer thickness $\delta$ in the following manner:

$\frac{\delta}{\delta_{t h}} \sim \mathrm{g}(\operatorname{Pr}, B n, n)$

where $g(\operatorname{Pr}, B n, n)$ is a function of $\operatorname{Pr}, B n$ and behavior index $(n)$. To estimate the hydrodynamic boundary layer thickness $\delta$, a balance of inertial and viscous forces in the vertical direction is considered:

$\rho\left[\frac{v^{2}}{L}\right] \sim \frac{\tau}{\delta}$

where $v$ is a characteristic velocity scale $(v \sim \sqrt{g \beta \Delta T L})$. For Herschel-Bulkley fluids the shear stress $\tau$ can be estimated as $\tau \sim \tau_{y}+\mu\left(\frac{v}{\delta}\right)^{2}$ which upon substitution in Eq. (27) gives:

$\rho\left[\frac{v^{2}}{L}\right] \sim\left[\tau_{y}+\mu\left(\frac{v}{\delta}\right)^{2}\right] \frac{1}{\delta}$

Using Eq. (27) and (28), the hydrodynamic boundary layer thickness can be estimated from the equation:

$f(\delta)=\delta^{n+1}\left[\frac{1}{L} \sqrt{\frac{R a}{P r}}\right]-B n \cdot \delta^{n}-B n \cdot \frac{\mu}{\tau_{y}} v^{n} \sim 0$

Stoer and Bulirsch [33] proposed a modification of the Newton method to estimate the first approximation of a root of a polynomial according to:

$\delta \sim \delta_{0}-\frac{f^{\prime}\left(\delta_{0}\right) \pm \sqrt{\left(f^{\prime}\left(\delta_{0}\right)\right)^{2}-2 f\left(\delta_{0}\right) f^{\prime \prime}\left(\delta_{0}\right)}}{f^{\prime \prime}\left(\delta_{0}\right)}$

$\delta_{0}$ is a proof ordinate value chosen as $\left(\delta_{0}=L \cdot \sqrt{\frac{P r}{R a}} B n\right)$, a relatively close value to the minimum $\left(\delta_{\min }=L \frac{n}{n+1} \sqrt{\frac{P r}{R a}} B n\right)$ of the function $f(\delta)$ with:

After evaluating $\delta_{0}$ in the equation (30) and replacing $\delta$ in equation (27), the hydrodynamic boundary layer thickness can be estimated as:

$\delta \sim \frac{L}{\Omega}\left\{\frac{2 n-1}{2 n} B n+\frac{1}{2 n}\left[\left(2 n^{3}-2 n^{2}+1\right) B n^{2}+4 n \frac{B n^{2-n}}{B n_{H B}}\left(\frac{R a}{P r}\right)^{n / 2}\right]^{1 / 2}\right\}$

where $B n_{H B}$ is the modified Bingham number for a HerschelBulkley fluid.

$B n_{H B}=\frac{\tau_{y}}{\mu}\left(\frac{L}{v}\right)^{n}$

This scaling gives rise to the following expression for the thermal boundary:

$\delta_{t h} \sim \min \left[L, \frac{1}{g(P r, B n, n)} \frac{L}{\Omega}\left\{\frac{2 n-1}{2 n} B n+\frac{1}{2 n}\left[\left(2 n^{3}-2 n^{2}+1\right) B n^{2}+4 n \frac{B n^{2}-n}{B n_{H B}}\left(\frac{R a}{P r}\right)^{n / 2}\right]^{1 / 2}\right\}\right]$

Substitution of Eq. (33) into Eq. (25) yields:

$\overline{N u} \sim \max \left[1.0, \frac{\sqrt{R a / P r}}{\left\{\frac{2 n-1}{2 n} B n+\frac{1}{2 n}\left[\left(2 n^{3}-2 n^{2}+1\right) B n^{2}+4 n \frac{B n^{2-n}}{B n_{H B}}\left(\frac{R a}{P r}\right)^{n / 2}\right]^{1 / 2}\right\}} g(P r, B n, n)\right]$

The expression (34) provides a useful insight into the expected behavior of $\mathrm{Nu}$ in response to variations of $\mathrm{Ra}, \mathrm{Pr}$, $B n$, and $n$. The scaling analysis suggests that $N u$ is expected to decrease with increasing $B n$ for given values of $R a$ and n, whereas $\mathrm{Nu}$ increases with increasing $\mathrm{Ra}$ for a given value of $B n$ and $\mathrm{n}$. For constant $\mathrm{Ra}, \mathrm{Pr}$, and $\mathrm{Bn}$, the effect of $\mathrm{n}$ on $\mathrm{Nu}$ is remarkable for shear-thinning fluids $(0<n<1)$ meanwhile for shear thickening fluids the effect is negligible. For $n=1$, the same scaling expression for $\mathrm{Nu}$ presented by Turan et al. [13] 
is obtained:

$\overline{N u} \sim \max \left[1.0, \frac{R a^{1 / 2} / \operatorname{Pr}^{1 / 2}}{\left\{\frac{1}{2} B n+\frac{1}{2}\left[B n^{2}+4\left(\frac{R a}{P r}\right)^{1 / 2}\right]^{1 / 2}\right\}} f(P r, B n)\right]$

\section{RESULTS AND DISCUSSIONS}

\subsection{Benchmark comparison and mesh independency study}

To verify the implemented numerical code and the pressure-velocity coupling algorithm (PRIME), the wellknown de Vahl Davis [34] benchmark problem of Newtonian natural convection process is replicated. The verification was performed for Rayleigh numbers ranging from $10^{3}$ to $10^{5}$ and Prandtl number corresponding to air, equal to $P r=0.71$ (Table 2 ). It should be mentioned here that because of the used reference value of velocity $(\sqrt{g \beta \Delta T L})$, the obtained velocity values must be multiplied by $\sqrt{\operatorname{RaPr}}$ compare with the Benchmark results. For the finer used mesh $(81 \times 81)$, the relative errors do not exceed $1 \%$ for the Nusselt and the maximum velocity values of $\mathrm{U}(\mathrm{Y}=0.5)$ and $\mathrm{V}(\mathrm{X}=0.5)$.

Table 2. Comparison of obtained results with the Benchmark for $\operatorname{Pr}=0.71$

\begin{tabular}{|c|c|c|c|c|c|}
\hline & & $\overline{N u}$ & $N u_{\max }$ & $\begin{array}{c}U_{\max } \\
(\mathrm{Y}=0.5)\end{array}$ & $\begin{array}{c}V_{\max } \\
(\mathrm{X}=0.5) \\
\end{array}$ \\
\hline & $31 \times 31$ & 1.098 & 1.506 & 3.627 & 3.691 \\
\hline \multirow[t]{2}{*}{$R a=10^{3}$} & $61 \times 61$ & 1.102 & 1.466 & 3.650 & 3.696 \\
\hline & $81 \times 81$ & 1.116 & 1.512 & 3.650 & 3.700 \\
\hline \multicolumn{2}{|c|}{ Benchmark } & 1.118 & 1.505 & 3.649 & 3.697 \\
\hline & $31 \times 31$ & 2.168 & 3.524 & 16.033 & 19.567 \\
\hline \multirow[t]{2}{*}{$R a=10^{4}$} & $61 \times 61$ & 2.209 & 3.532 & 16.130 & 15.583 \\
\hline & $81 \times 81$ & 2.222 & 3.538 & 16.172 & 19.632 \\
\hline \multicolumn{2}{|c|}{ Benchmark } & 2.243 & 3.528 & 16.178 & 19.617 \\
\hline & $31 \times 31$ & 4.346 & 7.649 & 33.614 & 69.603 \\
\hline \multirow[t]{2}{*}{$R a=10^{5}$} & $61 \times 61$ & 4.439 & 7.721 & 34.682 & 68.779 \\
\hline & $81 \times 81$ & 4.465 & 7.733 & 34.740 & 68.684 \\
\hline \multicolumn{2}{|c|}{ Benchmark } & 4.519 & 7.717 & 34.730 & 68.590 \\
\hline
\end{tabular}

Table 3. Comparison of corrected obtained results for Bingham fluid results with the Vola et al. [19] for $R a=10^{5}$, $\operatorname{Pr}=1.0$ and $B n=0.95$

\begin{tabular}{ccccc}
\hline & $\overline{N u}$ & $N u_{\max }$ & $\begin{array}{c}U_{\max } \\
(\mathrm{Y}=0.5)\end{array}$ & $\begin{array}{c}V_{\max } \\
(\mathrm{X}=0.5)\end{array}$ \\
\hline $\begin{array}{c}\text { Vola et al. } \\
(13)\end{array}$ & 4.0127 & 6.937 & 26.375 & 52.350 \\
$21 \times 21$ & 3.638 & 7.289 & 24.035 & 49.919 \\
$41 \times 41$ & 3.835 & 7.097 & 24.018 & 50.402 \\
$81 \times 81$ & 3.919 & 7.199 & 24.000 & 50.688 \\
\hline
\end{tabular}

The mesh independency study was performed for constant Rayleigh number and Prandtl number values (Pr=1.0; $R a=10^{5}$ ) considering a non-Newtonian Bingham fluid $(B n=0.95)$. According to the definition of the Bingham number (15b), this situation is equivalent to that made by Vola et al. [19] for a constant yield stress $\left(\tau_{y}=300 \mathrm{~Pa}\right)$. A comparison of the obtained values for the Nusselt number and maximum values of $\mathrm{U}(\mathrm{Y}=0.5)$ and $\mathrm{V}(\mathrm{X}=0.5)$ is shown in Table 3.

For a non-uniform mesh of $81 \times 81$ nodes, the absolute error does not exceed $3 \%$. Then, according to this and to the deviation of the obtained results respect to the benchmark results, a mesh of $81 \times 81$ nodes is used during the calculations with $R a=10^{5}$, and a mesh of $61 \times 61$ (Figure 3) nodes is used. Non-uniform meshes with a higher density of elements near to the enclosure walls are used since the larger velocity, and temperature gradients take place in these zones.

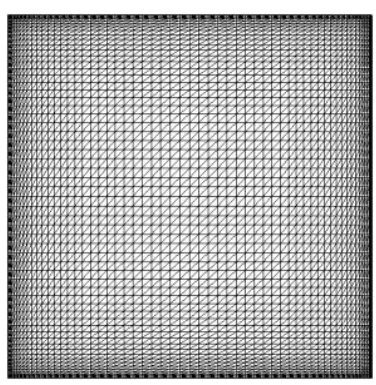

Figure 3. Non-uniform mesh used with 3721 nodes and 7200 elements for cases with $R a<10^{5}$

\subsection{Natural convection heat transfer behavior}

The natural convection heat transfer process of a HerschelBulkley fluid is studied by varying the dimensionless parameters: $\operatorname{Pr}, \operatorname{Ra}, \mathrm{Bn}$ and $n$. Table 4 shows the Nusselt numbers obtained for $R a=10^{3}, R a=10^{4}$ and $R a=10^{5}$ as a function of the Bingham and Prandtl numbers for a constant behavior index $(n=0.7)$.

Table 4. Nusselt numbers for a Herschel-Bulkley fluid $(n=0.7)$

\begin{tabular}{cccccccc}
\hline$B n$ & \multicolumn{2}{c}{0.01} & & 0.1 & & \multicolumn{2}{c}{1} \\
\hline$P r / R a$ & $10^{3}$ & $10^{4}$ & $10^{3}$ & $10^{4}$ & $10^{5}$ & $10^{3}$ & $10^{4}$ \\
0.1 & 1.16 & $\underline{2.31}$ & 1.11 & $\underline{2.29}$ & - & - & $\underline{2.02}$ \\
1 & 1.06 & 2.30 & 1.03 & 2.21 & 4.99 & 1.01 & 1.41 \\
2 & - & - & - & - & 4.87 & - & - \\
5 & - & - & - & - & 4.59 & - & - \\
10 & 1.02 & 1.96 & 1.00 & 1.72 & 4.35 & 1.00 & 1.01 \\
\hline
\end{tabular}

According to these results, the Nusselt number is a strong function of the $R a$ (see values in bold), while $N u$ is a weaker function of the $\operatorname{Pr}$ (see values in cursive) and $B n$ (see underlined values). As predicted by the scaling analysis, the $N u$ number increases with the $R a$ number, and it decreases as the $P r$ and $B n$ numbers increases. When the $B n$ number rises the fluid has higher yield stress; therefore the convection is depleted, and heat transfer by conduction becomes more important. In Figure 4 can be seen how the numerical experiment conducted at a low Rayleigh number $\left(R a=10^{3}\right)$. It allows observing the effect of the $B n$ and $P r$ numbers in the diffusion heat transfer mode. When the $B n$ and $P r$ numbers are lower than 0.01 and 0.1 , respectively, the effect of the convective heat transfer starts to be noticeable since the isotherms adopt a sigmoidal shape. When the $P r$, therefore the $G r$ number becomes lower, and Bingham numbers become higher, conduction is the more important heat transfer mechanism, and the isotherms are almost parallel to the isothermal surfaces, as expected. The distribution of isotherms in Figure 5 shows the importance of the buoyancy forces on the heat transfer process when the Rayleigh number is increased $\left(R a=10^{4}\right)$. In this set of results, it can be seen the effect of heat transfer by conduction when the Prandtl and Bingham numbers increase $(P r=10$ and $B n=1.0)$, that is when the fluid transfers momentum at a higher rate than energy and 
the yield strength becomes higher than the viscosity of the fluid, or when the fluid tends to be a solid material.

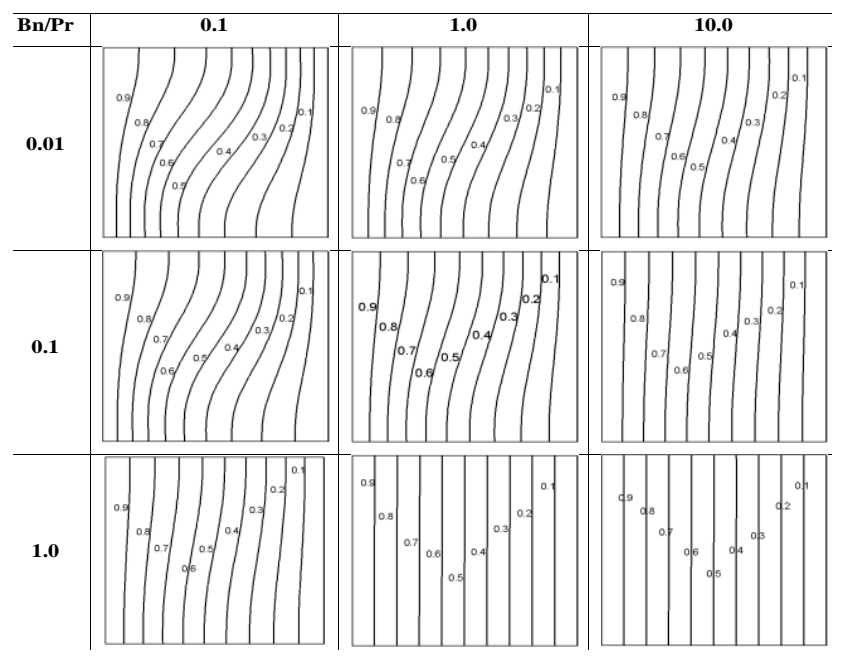

Figure 4. Effect of $\operatorname{Pr}$ and $B n$ numbers on isotherms for $R a=10^{3}$ and $\mathrm{n}=0.7$

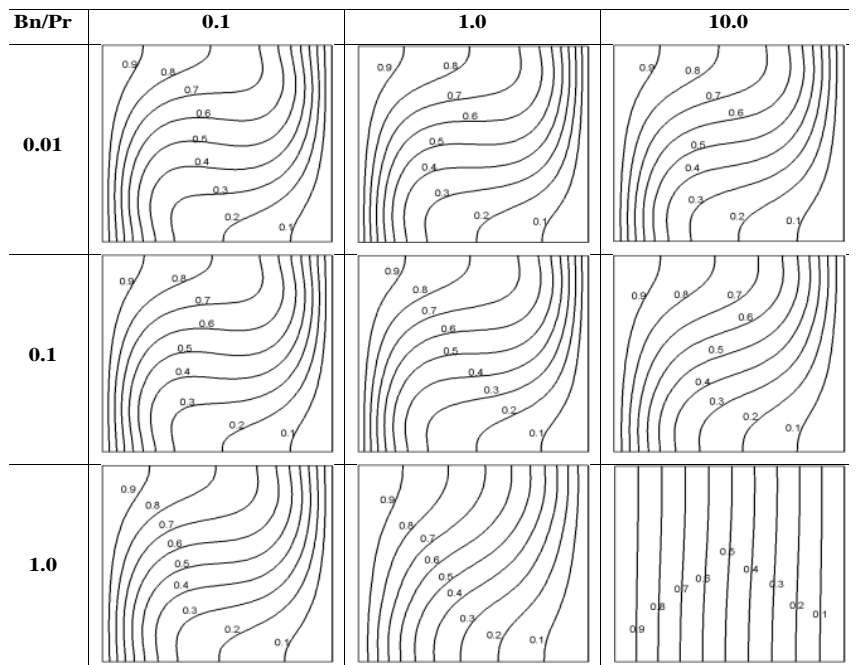

Figure 5. Effect of $\operatorname{Pr}$ and $B n$ numbers on isotherms for $R a=10^{4}$ and $\mathrm{n}=0.7$

In order to increase the comprehension of the behavior of the Herschel-Bulkley fluids, an analysis of the behavior of the fluid as a function of the dimensionless numbers $R a, P r$ and $B n$ is performed. When the Rayleigh number is low $\left(R a=10^{3}\right)$, the streamlines are concentric, adopting a rounded shape near to the center of the cavity, and taking the form of the walls as they move away from the center (Figure 6). In this case, only one central vortex can be seen without the presence of secondary flows, which in two-dimensional domains are evidenced by the presence of smaller vortices near to the lower corners of the cavity. The absence of these vortices is explained by zones of high viscosity $(H>10)$, where the yield value is not exceeded (unyielded zone demarcated in red in the same figure). When the Bingham number increases, from 0.01 to 0.1 , the unyielded zones appear close the center of the cavity, breaking the "natural convection motor" and promoting the heat conduction. It is evident that the unyielded zones have a symmetrical distribution around the center of the vortex when the conduction is the more important heat transfer regime $(\operatorname{Pr}=10, B n=0.1$, and $\operatorname{Pr}=1.0, B n=1)$.

In Figure 7, the streamlines that describe the behavior of the fluid mechanics for a higher Rayleigh number $\left(R a=10^{4}\right)$ are shown. In general, the streamlines are similar to those observed for $R a=10^{3}$. However, when the $\operatorname{Pr}$ and $B n$ numbers are increased, the concentric fluid trajectories appear more flattened and squared, respectively. The higher viscosity regions, demarcated in red in the same figure, that occupy a broader region in the cavity are found when $B n=0.1$ and $B n=1.0$, for $P r=10$ and $P r=1.0$; respectively. However, the effect of conduction on heat transfer becomes important only in the latter case (Figure 5).

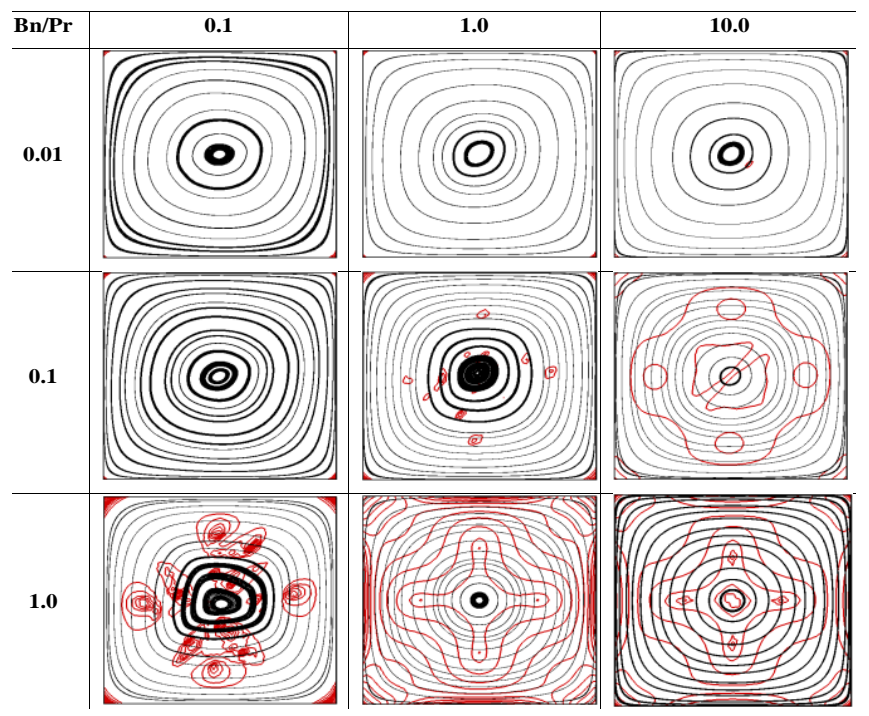

Figure 6. Effect of $P r$ and $B n$ numbers on streamlines for $R a=10^{3}$ and $n=0.7$. The red zones correspond to unyielded regions in the cavity

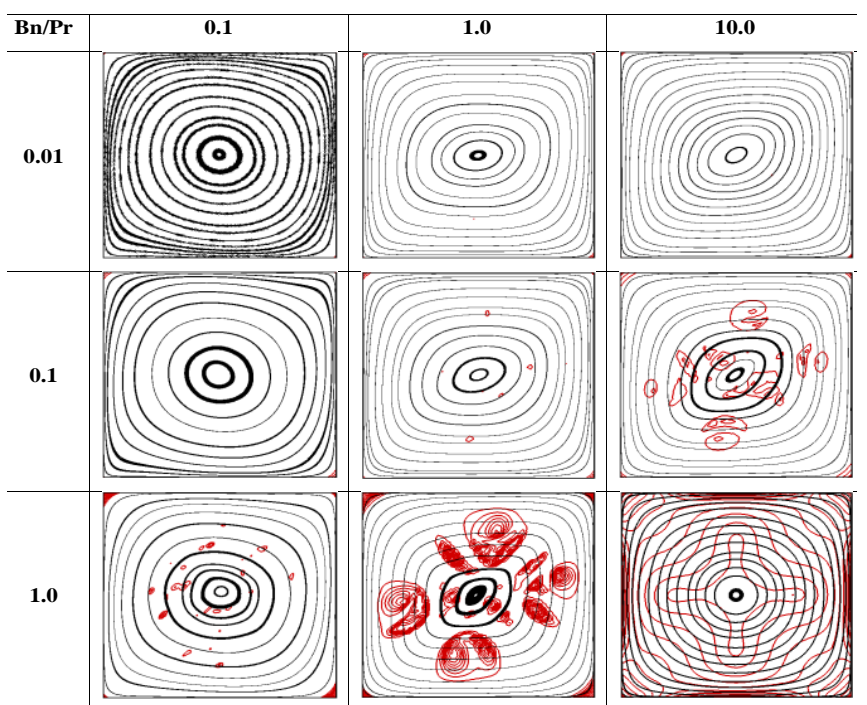

Figure 7. Effect of $P r$ and $B n$ numbers on streamlines for $R a=10^{4}$ and $n=0.7$. The red zones correspond to unyielded regions in the cavity

A study of the vertical component of the velocity $\mathrm{V}$ along the axis $\mathrm{X}$ for $\mathrm{Y}=0.5$ (Figure 8) is added to the previous descriptive analysis. When the Prandtl number is increased by one order of magnitude, from $\operatorname{Pr}=0.1$ to $\operatorname{Pr}=1$ for a constant Bingham number, the velocity decreases by the same order of magnitude. However, this depletion is more pronounced when the Rayleigh number is lower $\left(R a=10^{3}\right)$, and the Prandtl number is higher $(\operatorname{Pr}=10)$. For $R a=10^{4}$, when the Prandtl 
number is increased from 0.1 to 1.0 , the velocity is decreased about one order of magnitude, but a further increase from 1.0 to 10 does not change the velocity profile substantially. The Bingham number has not an important effect on the velocity distribution, when $\mathrm{Pr}$ is constant. This observation is more evident when the yield strength becomes as important as the viscous forces $(B n=1)$, and the number Rayleigh is lower $\left(R a=10^{3}\right)$.

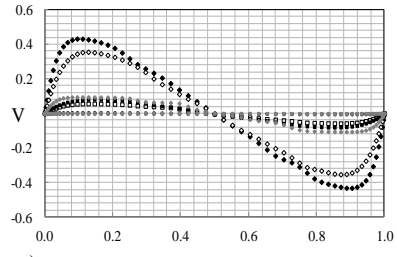

a)

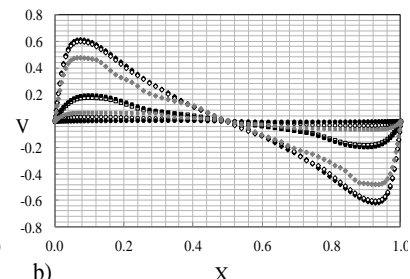

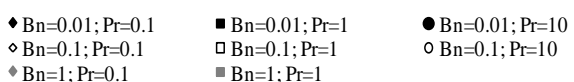

Figure 8. Vertical component of velocity $\mathrm{V}$ along the horizontal axis $\mathrm{X}\left(\mathrm{Y}=0.5\right.$ ) for a) $R a=10^{3}$ and b) $R a=10^{4}$ with $\mathrm{n}=0.7$

For a constant Bingham number, the increasing of the Rayleigh number to $R a=10^{5} \quad(P r=0.1)$ makes the heat convection more important than the heat conduction. In Figure 9, this effect is noticed by observing the sigmoidal shape of the isotherms. From the qualitative point of view, the effect of the Prandtl number on the isotherms distribution is not important, except in those points where the velocity gradient has a pronounce change, some of them are highlighted in Figure 9, especially those close to the wall at a lower temperature $(\mathrm{X}=\mathrm{L})$. The behavior of the streamlines predicts the existence of two vortices in the center of the cavity, slightly displaced when $\operatorname{Pr}=1$, which tend to be flattened and fused when the Prandtl number rises. The areas of high viscosity $(H>10)$ appear in a region of high intensity of the streamlines and in the upper left and lower right of the cavity.

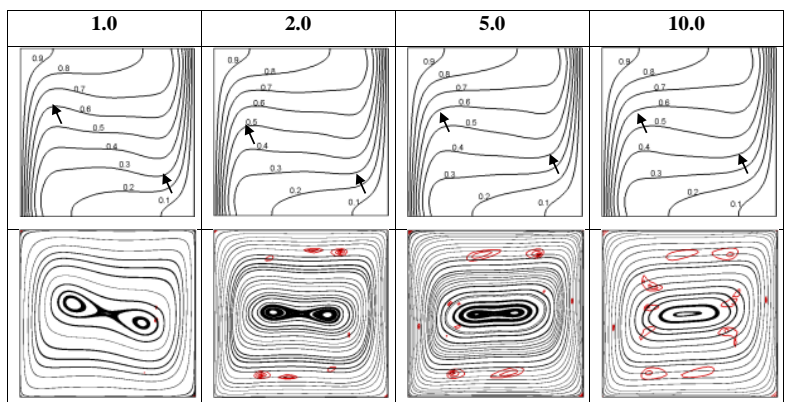

Figure 9. Effect of the $P r$ number on isotherm and streamlines for $R a=10^{5}, B n=0.1$ and $\mathrm{n}=0.7$

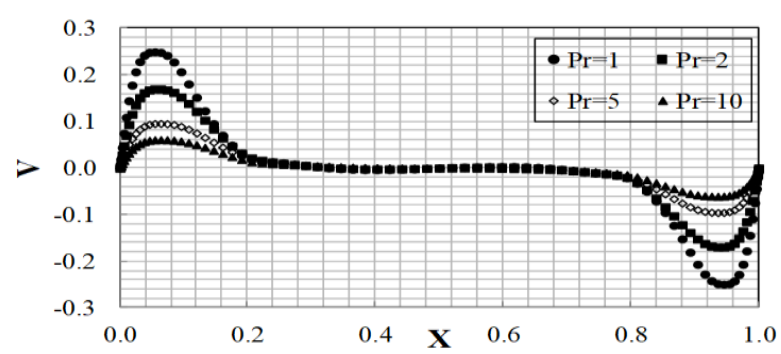

Figure 10. Vertical component of velocity along the horizontal axis $\mathrm{X}(\mathrm{Y}=0.5)$ for $R a=10^{5}, B n=0.1$ and $\mathrm{n}=0.7$
In Figure 10, the effect of the Prandtl number on the Ycomponent of velocity profile along the $\mathrm{X}$-axis $(\mathrm{Y}=0.5)$ is shown. The observed shape of the velocity profile is typical of the natural convection processes with a high Grashof number. When the Prandtl number becomes higher the maximum velocity value close to the walls drops as well as the velocity gradient, since a decrease of the shear stress on the walls is expected. The Herschel-Bulkley model is characterized by an exponential term called the behavior index $(n)$. When $n=1$ the Hershel-Bulkley models becomes the Bingham model. The effect of the behavior index is studied in the range of 0.7 to 1.5 .

Figure 11 shows the distribution of isotherms within the cavity for different behavior index values. From the qualitative point of view, no significant differences are observed. A deeper insight into the effect of the behavior index is given by observing the Nusselt number. The analysis of the variation of the Nusselt number with the behavior index (Figure 12) confirms the prediction made by the scaling analysis. With $R a$, $P r$ and $B n$ as parameters, the increase of the behavior index causes a decrease of the effect of convection in the heat transfer process (lower Nusselt number), being this effect more important when the behavior is in the range $0<n<1$.

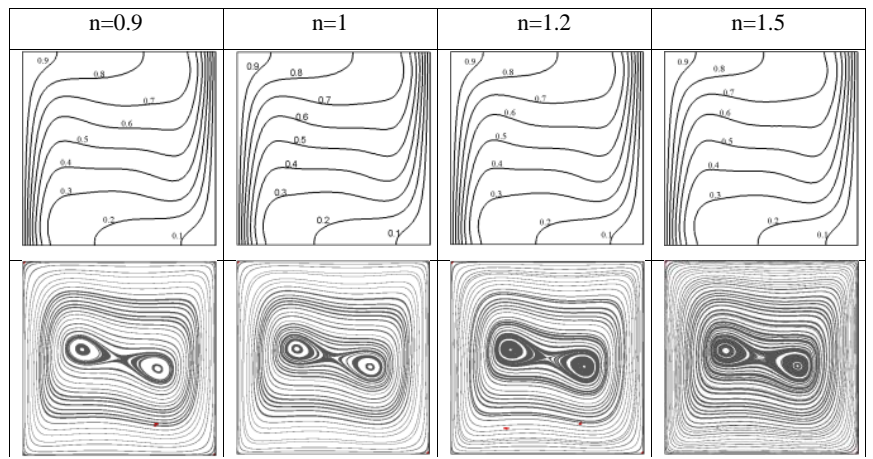

Figure 11. Effect of the behavior index (n) on streamlines and isotherms for $R a=10^{5}$ and $\mathrm{Bn}=0.1$

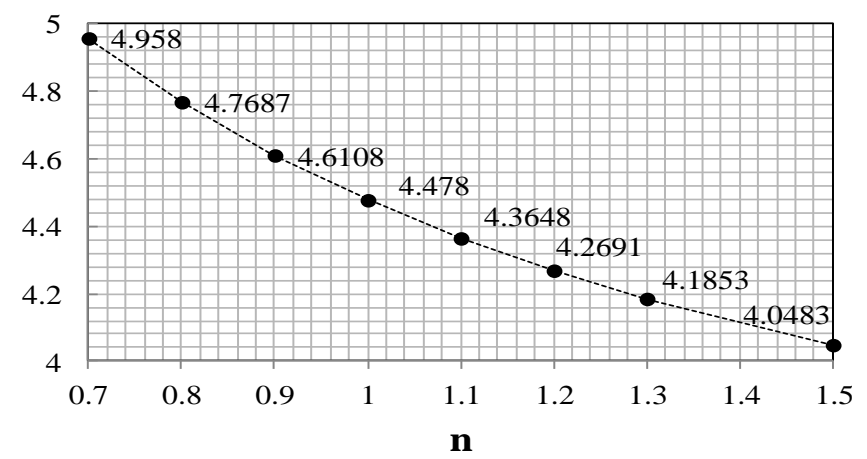

Figure 12. Nusselt number variation as a function of the behavior index (n) for $R a=10^{5}$ and $B n=0.1$

The effect of the behavior index in the streamlines is not relevant from the qualitative point of view (Figure 11). The positions of the vortex in the center of the cavity are equally displaced from the central axis $(\mathrm{Y}=0.5)$. In the Figure 13, the effect of the behavior index in the $\mathrm{Y}$-velocity profile along the axis $\mathrm{X}$ is shown. A detailed view allows distinguishing the larger variation of the velocity profile near to the isothermal walls of the cavity. It is observed that there is no a single trend. Higher Y-velocities near to the wall are observed when $n<1$ 
and after certain critical position $(\mathrm{X} \sim 0.1)$ the $\mathrm{Y}$-velocities values are higher for $n>1$.

The behavior index $\mathrm{n}$ has an important effect on the computational time. For the smallest studied value $(n=0.7)$ the CPU time is higher $(360 \mathrm{~h})$ than for $n=1(270 \mathrm{~h})$. For intermediate values $0.8<\mathrm{n}<1.5$ the CPU times are of the same $\operatorname{order}(270 \mathrm{~h})$ when $n=1$. If the behavior index is higher $(\mathrm{n}=2)$, the CPU time is significantly lower $(100 \mathrm{~h})$ than for $n=1$. These differences can be explained by the relative weight of the diffusive term with respect to the convective term in the Navier-Stokes equation. When the strain rate is more significant than one and $n<1$, the relative weight given to the diffusive term is lower, and therefore the convective term becomes more important. The opposite occurs when $n>1$.
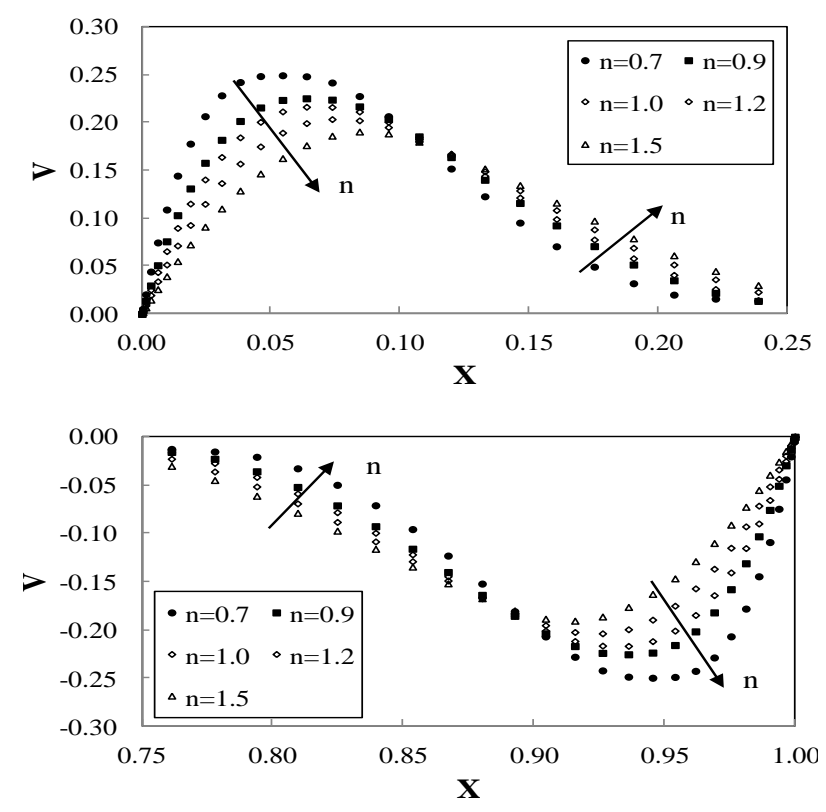

Figure 13. Effect of the behavior index (n) in the $\mathrm{Y}$-velocity profile along the horizontal axis $(\mathrm{Y}=0.5)$ for $R a=10^{5}$ and $B n=0.1$

\section{CONCLUSIONS}

A developed code based on CVFEM was implemented to solve natural convection heat transfer in a cavity for a Herschel-Bulkley non-Newtonian fluid. The performed scaling analysis allowed a preliminary identification of the importance of $R a, P r, B n, \mathrm{n}$, and Nu numbers. The $R a$ number had a sigificant effect on both heat transfer and fluid mechanics. The effect of the $P r$ and $B n$ is more important when the Ra number is low $\left(10^{3}\right)$. When the $\operatorname{Pr}(10)$ and $B n(1.0)$ numbers are increased, the heat transfer becomes a diffusive process, because of the reduced effect of convection and the increased influence of the yield stress, respectively.

Also, the increasing of the behavior index causes a decrease in the Nu number. This effect was more important when $\mathrm{n}<1$. The analysis of the vertical component of the velocity (V) along the horizontal axis (X) allows identifying a change in the trend of the velocity profiles. Close to the isothermal walls, $\mathrm{V}$ decreases when the behavior index was increased, and this trend was reversed after a critical point, located around $\mathrm{X}=0.1$ and $\mathrm{X}=0.95$.

The behavior index had an effect on the CPU time. For one of the studied cases $\left(R a=10^{5}, B n=0.1, P r=1\right)$, when the behavior index is the lower $(n=0.7)$ the computation time is $33 \%$ greater than for the Bingham fluid $(n=1)$. For intermediate values $0.8<\mathrm{n}<1.5$ the $\mathrm{CPU}$ time was of the same order of magnitude than when the fluid was of the Bingham type $(n=1)$. When the performance index was larger, $n=2$, the CPU time was much lower $(63 \%$ over $n=1)$. These differences were explained by the different relative weight of the diffusion concerning to the convective terms in the Navier-Stokes equations.

\section{ACKNOWLEDGMENT}

This work was funded by CONICYT/Chile under Fondecyt Project 11130168

\section{REFERENCES}

[1] Barnes H. (1999). The yield stress-a review-everything flows? Journal of Non-Newtonian Fluid Mechanics 81(1-2): 133-178. https://doi.org/10.1016/S03770257(98)00094-9

[2] Zhu H, Kim Y, De Kee D. (2005). Non-Newtonian fluids with a yield stress. Journal of Non-Newtonian Fluid Mechanics 129(3): 177-181. https://doi.org/10.1016/j.jnnfm.2005.06.001

[3] Tabilo-Munizaga G, Barbosa-Cánovas G. (2005). Rheology for the food industry. Journal of Food Engineering 67(1-2): 147-156. https://doi.org/10.1016/j.jfoodeng.2004.05.062

[4] Gratão A, Silveira Jr.V, Telis-Romero J. (2007). Laminar flow of soursop juice through concentric annuli: Friction factors and rheology. Journal of Food Engineering 78(4): 1343-1354. https://doi.org/10.1016/j.jfoodeng.2006.01.006

[5] Trigilio-Tavares D, Alcantara M, Tadini C, TelisRomero J. (2007). Rheological properties of frozen concentrated orange juice (FCOJ) as a function of concentration and subzero temperatures. International Journal of Food Properties 10(4): 829-839. https://doi.org/10.1080/10942910601118805

[6] Telis-Romero J, Telis V, Yamashita F. (1999). Friction factors and rheological properties of orange juice. Journal of Food Engineering 40(1-2): 101-106. https://doi.org/10.1016/S0260-8774(99)00045-X

[7] Vandresen S, Quadri M, de Souza J, Hotza D. (2009). Temperature effect on the rheological behavior of carrot juices. Journal of Food Engineering 92(3): 269-274. https://doi.org/10.1016/j.jfoodeng.2008.11.010

[8] Leong W, Hollands K, Brunger A. (1998). On a physically-realizable benchmark problem in internal natural convection. International Journal of Heat and Mass Transfer 41(23): 3817-3828. https://doi.org/10.1016/S0017-9310(98)00095-7

[9] Leong W, Hollands K, Brunger A. (1999). Experimental Nusselt numbers for a cubical-cavity benchmark problem in natural convection. International Journal of Heat and Mass Transfer 4(11): 1979-1989. https://doi.org/10.1016/S0017-9310(98)00299-3

[10] Fusegi T, Hyun J, Kuwahara K, Farouk B. (1991). A numerical study of three-dimensional natural convection in a differentially heated cubical enclosure. International Journal of Heat and Mass Transfer 34(6): 1543-1557. https://doi.org/10.1016/0017-9310(91)90295-P 
[11] Wen-Hann ST, Reui-Kuo LR-K. (2011). Threedimensional bifurcations in a cubic cavity due to buoyancy-driven natural convection. International Journal of Heat and Mass Transfer 54(1-3): 447-467. https://doi.org/10.1016/j.ijheatmasstransfer.2010.09.024

[12] O'Donovan E, Tanner R. (1984). Numerical study of the Bingham squeeze film problem. Journal of NonNewtonian Fluid Mechanics 15(1): 75-83. https://doi.org/10.1016/0377-0257(84)80029-4

[13] Turan O, Chakraborty N, Pool R. (2010). Laminar natural convection of Bingham fluids in a square enclosure with differentially heated side walls. Journal of Non-Newtonian Fluid Mechanics 165(15-16): 901-913. https://doi.org/10.1016/j.jnnfm.2010.04.013

[14] Khalifeh A, Clermont J. (2005). Numerical simulations of non-isothermal three-dimensional flows in an extruder by a finite-volume method. Journal of Non-Newtonian Fluid Mechanics 126(1): 7-22. https://doi.org/10.1016/j.jnnfm.2004.12.002

[15] Barth W, Carey G. (2006). On a natural-convection benchmark problem in non-Newtonian fluids. Numerical Heat Transfer, Part B: Fundamentals 50(3): 193-216. https://DOI: 10.1080/10407790500509009.

[16] Anwar-Hossain M, Reddy-Gorla R. (2009). Natural convection flow of non-Newtonian power-law fluid from a slotted vertical isothermal surface. International Journal of Numerical Methods for Heat \& Fluid Flow 19(7):

835-846. https://doi.org/10.1108/09615530910984109

[17] Lamsaadi M, Naïmi M. (2006). Natural convection in a vertical rectangular cavity filled with a non-Newtonian power law fluid and subjected to a horizontal temperature gradient. Numerical Heat Transfer, Part A: Applications 49(10): 969-990. https://doi.org/10.1080/10407780500324988

[18] Lamsaadi M, Naïmi M, Hasnaoui M. (2006). Natural convection heat transfer in shallow horizontal. Energy Conversion and Management 47(15-16): 2535-2551. https://doi.org/10.1016/j.enconman.2005.10.028

[19] Vola D, Boscardin L, Latché J. (2003). Laminar unsteady flows of Bingham fluids: a numerical strategy and some benchmark results. Journal of Computational Physics 187(2): 441-456. https://doi.org/10.1016/S00219991(03)00118-9

[20] Siginer D, Valenzuela-Rendon A. (2000). On the laminar free convection and stability of grade fluids in enclosures. International Journal of Heat and Mass Transfer 43(18): 3391-3405. https://doi.org/10.1016/S0017-9310(99)00357-9

[21] Kumar A, Bhattacharya M. (1991). Transient temperature and velocities profiles in a canned nonNewtonian liquid food during sterilization in a still-cook retort. International Journal of Heat and Mass Transfer 34(4-5): 1083-1096. https://doi.org/10.1016/00179310(91)90018-A

[22] Kim G, Hyun J, Kwak H. (2003). Transient buoyant convection of a power-law non-Newtonian fluid in an enclosure. International Journal of Heat and Mass Transfer 46(19): 3605-3617. https://doi.org/10.1016/S0017-9310(03)00149-2

[23] Dean E, Glowinski R, Guidoboni G. (2007). On the numerical simulation of Bingham visco-plastic flow: Old and new results. Journal of Non-Newtonian Fluid
Mechanics.

1421-3)

$36-62$.

https://doi.org/10.1016/j.jnnfm.2006.09.002

[24] Baliga B, Patankar S. (1980). A new finite element formulation for convection diffusion problems. Numerical Heat Transfer 3(4): 393-409. https://doi.org/10.1080/01495728008961767

[25] Voller V. (2009). Basic control volume finite element methods for fluid and solids, IISC Press, Singapore.

[26] Silva J, De Moura, L. (2001). A control-volume finiteelement method (CVFEM) for unsteady, incompressible, viscous fluid flows. Numerical Heat Transfer, Part B: Fundamentals 40(1): 61-82. https://doi.org/10.1080/104077901300233604

[27] Salinas C, Chavez C, Gatica Y, Ananias R. (2011). Simulation of wood drying stresses using CVFEM. Latin American Applied Research 41(1): 23-30.

[28] Estacio K, Nonato L, Mangiavacchi N, Carey G. (2008). Combining CVFEM and meshless front tracking in Hele-Shaw mold filling simulation. International Jouurnal of Numerical Methods in Fluid. 56: 1217-1223. https://doi.org/10.1002/fld.1732

[29] Charoensuk J, Vessakosol P. (2010). A high order control volume finite element procedure for transient heat conduction analysis of functionally graded materials. Heat and Mass Transfer 46(11-12): 12611276. https://DOI 10.1007/s00231-010-0649-8.

[30] Herschel W, Bulkley R, (1926). Konsistenzmessungen von Gummi-Benzollösungen. Colloid Polym Sci. 39: 291-300.

[31] Papanastasiou T. (1987). Flows of materials with yield. Journal of Rheology 31(5): 385-404. https://doi.org/10.1122/1.549926

[32] Abdali S, Mitsoulis E, Markatos N. (1992). Entry and exit flows of Bingham fluids. Journal of Rheology 36: 389-407. https://doi.org/10.1122/1.550350

[33] Stoer J, Bulirsch R. (1980). Introduction to numerical analysis, Springer, New York, USA.

[34] Vahl Davis G. (1983). Natural convection of air in a square cavity: A bench mark numerical solution. International Journal for Numerical Methods in Fluids 3: 249-264. https://doi.org/10.1002/fld.1650030305

\section{NOMENCLATURE}

$\begin{array}{ll}A, B, C & \text { Linear interpolation coefficients } \\ B n & \text { Bingham number, } B n=\tau_{y} / \mu(L /(g \beta \Delta T))^{0.5} \\ C p & \text { heat capacity, } J \mathrm{~kg}^{-1} \mathrm{~K}^{-1} \\ F & \text { Source term } \\ g & \text { gravitational acceleration, } \mathrm{m} \mathrm{s}^{-2} \\ G r & \text { Grashof number, } \mathrm{Gr}=g \beta \Delta T L^{3} / \eta_{o}^{2} \\ h & \text { convective coefficient, } W \mathrm{~m}^{-2} K^{-1} \\ \vec{J} & \text { interfacial flux } \\ \mathrm{K} & \text { consistency index, } P a \mathrm{~s}^{-n} \\ k & \text { thermal conductivity, } W \mathrm{~m}^{-1} K^{-1} \\ L & \text { characteristic dimensionless length } \\ m & \text { exponential growth parameter, s } \\ M & \text { exponential growth parameter } \\ n & \text { behavior index } \\ \hat{n} & \text { normal unitary vector } \\ \mathrm{Nu} & \text { Nusselt } \text { number, } N u=h L / K \\ p & \text { Pressure, } P a \\ P & \text { dimensionless pressure, } P=p L \eta_{o} / u_{o} \\ P e & \text { Peclet number, } P e=\rho U l / \mu\end{array}$




\section{Greek symbols}

$\alpha$
$\beta$
$\gamma$
$\delta$
$\zeta$
$\eta$
$H$
$\theta$

\section{$\alpha$}

$\beta$
Prandtl number, $\operatorname{Pr}=\eta_{o} \mathrm{Cp} / \mathrm{k}$

heat flux, $\mathrm{W} \mathrm{m} \mathrm{m}^{-2}$

Rayleigh number, $R a=g \beta \Delta T L^{3} / \eta_{o} \alpha$

contour area of a control volume, $\mathrm{m}^{2}$

time, $s$

temperature, $K$

velocity components, $m \mathrm{~s}^{-1}$

dimensionless velocity components,

$U=u / u_{o} ; V=v / u_{o}$

volume of a control volume, $\mathrm{m}^{3}$

velocity vector

lengths, $m$

dimensionless lengths, $X=x / L ; Y=y / L$

local coordinate parallel to the velocity vector

thermal diffusivity, $m^{2} s^{-1}$

thermal expansion coefficient, $K^{-1}$

deformation rate, $s^{-1}$

deformation rate tensor

dimensionless deformation rate, $\Gamma=L / u_{o} \gamma$

boundary layer thickness, $m$

difference between values

local coordinate perpendicular to the

velocity vector

apparent viscosity, $\mathrm{Pa} \mathrm{s}$

dimensionless apparent viscosity, $H=\eta / \eta_{o}$

dimensionless temperature, $\theta=\left(T-T_{C}\right) /\left(T_{H^{-}}\right.$

$\left.T_{C}\right)$ )

density, $\mathrm{kg} / \mathrm{m}^{-3}$

$\rho$

$\tau_{y}$

$\overline{\bar{\tau}}$

$\phi$

\section{Subscripts}

$a, b, c$

c

$e$

$H$

$k$

$i, j$

$\max$

$\min$

$n b$

$p$

ref

th

$o$

\section{Superscripts}

E

$k$

$l$

$n$

$N$ yield stress, $\mathrm{N} \mathrm{m}^{-2}$

shear stress tensor

dependent variable, $u, v, T$

vertices of a finite element

cold

corresponding value of a finite element

hot

contour of a control volume

Cartesian coordinate component

maximum value

minimum value

neighbor nodes

reference node

reference

thermal

reference value

total number of finite elements

iteration number

value of a control volume

value of a control volume

total number of control volumes 